\title{
Sac Angiography and Glue Embolization in Emergency Endovascular Aneurysm Repair for Ruptured Abdominal Aortic Aneurysm
}

\author{
Yuya Koike $\cdot$ Jun-ichi Nishimura $\cdot$ Soichiro Hase $\cdot$ \\ Motoshige Yamasaki
}

Received: 27 November 2013/Accepted: 25 January 2014/Published online: 14 March 2014

(C) Springer Science+Business Media New York and the Cardiovascular and Interventional Radiological Society of Europe (CIRSE) 2014

\begin{abstract}
Purpose The purpose of this study was to demonstrate a sac angiography technique and evaluate the feasibility of $\mathrm{N}$-butyl cyanoacrylate (NBCA) embolization of the ruptured abdominal aortic aneurysm (AAA) sac in emergency endovascular aneurysm repair (EVAR) in hemodynamically unstable patients.

Methods A retrospective case series of three patients in whom sac angiography was performed during emergency EVAR for ruptured AAA was reviewed. After stent graft deployment, angiography within the sac of aneurysm (sac angiography) was performed by manually injecting $10 \mathrm{ml}$ of contrast material through a catheter to identify the presence and site of active bleeding. In two patients, sac angiography revealed active extravasation of the contrast material, and NBCA embolization with a coaxial catheter system was performed to achieve prompt sealing.

Results Sac angiography was successful in all three patients. In the two patients who underwent NBCA embolization for aneurysm sac bleeding, follow-up computed tomography (CT) images demonstrated the accumulation of NBCA consistent with the bleeding site in preprocedural CT images.
\end{abstract}

\footnotetext{
Y. Koike $(\bowtie) \cdot$ J. Nishimura $\cdot$ S. Hase $\cdot$ M. Yamasaki

Department of Interventional Radiology, Kawasaki Saiwai

Hospital, 31-27, Omiya-cho, Saiwai-Ku, Kawasaki,

Kanagawa 212-0014, Japan

e-mail: r06118@hotmail.co.jp

J. Nishimura

e-mail: jun-ichi_n@nifty.com

S. Hase

e-mail: haseman@hotmail.co.jp

M. Yamasaki

e-mail: genyamasaki@gmail.com
}

Conclusions EVAR is associated with a potential risk of ongoing bleeding from type II or IV endoleaks into the disrupted aneurysm sac in patients with severe coagulopathy. Therefore, sac angiography and NBCA embolization during emergency EVAR may represent a possible technical improvement in the treatment of ruptured AAA in hemodynamically unstable patients.

Keywords Ruptured abdominal aortic aneurysm . Endovascular aneurysm repair $\cdot \mathrm{N}$-butyl cyanoacrylate

\section{Introduction}

Endovascular aneurysm repair (EVAR) of ruptured abdominal aortic aneurysms (r-AAAs) is feasible and offers the potential for decreased morbidity and mortality [1-3]. There also is currently an increased recognition of abdominal compartment syndrome [3]. Even after EVAR, ongoing bleeding from type II or IV endoleaks into the disrupted aneurysm sac in patients with severe coagulopathy may contribute to the size of the hematoma [3].

Therefore, we developed a procedure for direct sealing of the bleeding site in the aneurysm sac during emergency EVAR as follows:

1. Sac angiography after stent-graft deployment was performed to evaluate the presence or absence of active bleeding from the aneurysm sac.

2. When the active bleeding continued, embolization using $N$-butyl cyanoacrylate was performed.

We herein introduce this technique of sac angiography in emergency EVAR and demonstrate our early experience 


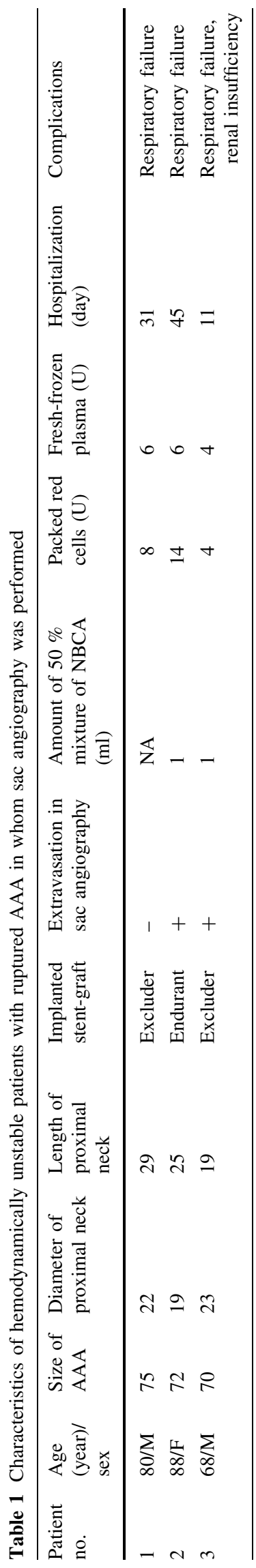

with glue embolization during emergency EVAR in hemodynamically unstable patients with r-AAA.

\section{Materials and Methods}

Between September 2012 and October 2013, three consecutive patients (two men, one woman; mean age, 78 years; range $68-88$ years) underwent sac angiography during emergency EVAR for r-AAA. All patients presented with abdominal pain and vital signs indicating hemodynamic instability. Hemodynamic instability was defined as loss of consciousness or a systolic blood pressure of $<80 \mathrm{~mm} \mathrm{Hg}$ despite ongoing resuscitation before induction of anesthesia. All patients were confirmed to have an r-AAA with active bleeding by contrast-enhanced computed tomography (CT). The average aneurysm diameter was 72 (range 70-75) $\mathrm{mm}$, the average proximal aneurysm neck length was 24 (range 19-29) $\mathrm{mm}$, and the proximal neck diameter was 21 (range 19-23) mm. Under our policy, the preferential treatment was EVAR. Open surgery was only selected as the treatment when anatomic criteria precluded effective exclusion of the aneurysm.

In this series, the Endurant Stent Graft System (Medtronic Cardiovascular, Santa Rosa, CA) was used in one patient, and the Excluder AAA Endoprosthesis (W. L. Gore \& Associates, Flagstaff, AZ) was used in two patients (Table 1).

\section{Procedure}

All endovascular procedures were performed in a hybrid operating room under fluoroscopy (Allura Xper FD20; Philips Medical Systems, Best, Netherlands). The procedures were started with percutaneous insertion of an aortic occlusion balloon catheter under local anesthesia as needed [4]. The patients then underwent general anesthesia and surgical exposure of their femoral arteries with or without aortic balloon occlusion.

Initially, the main body of the stent-graft was introduced through the contralateral access site (which was the opposite side of the occlusion balloon catheter) over a 0.035-inch stiff guidewire (Amplatz Extra-Stiff Wire; Cook Medical, Bloomington, IN) and deployed after the renal artery location was confirmed via aortography. The occlusion balloon catheter was then counterchanged through the main body side. After cannulation of the contralateral gate and exchange to a stiff wire, a 0.035 -inch standard J guidewire (Terumo, Tokyo, Japan) was advanced into the sac of the aneurysm (between the stentgraft and native aorta) via the same sheath $\left(18 \mathrm{~F}\right.$ Gore ${ }^{\circledR}$ DrySeal Sheath, W. L. Gore \& Associates) in parallel with the stiff wire (Fig. 1B). While maintaining the position of 


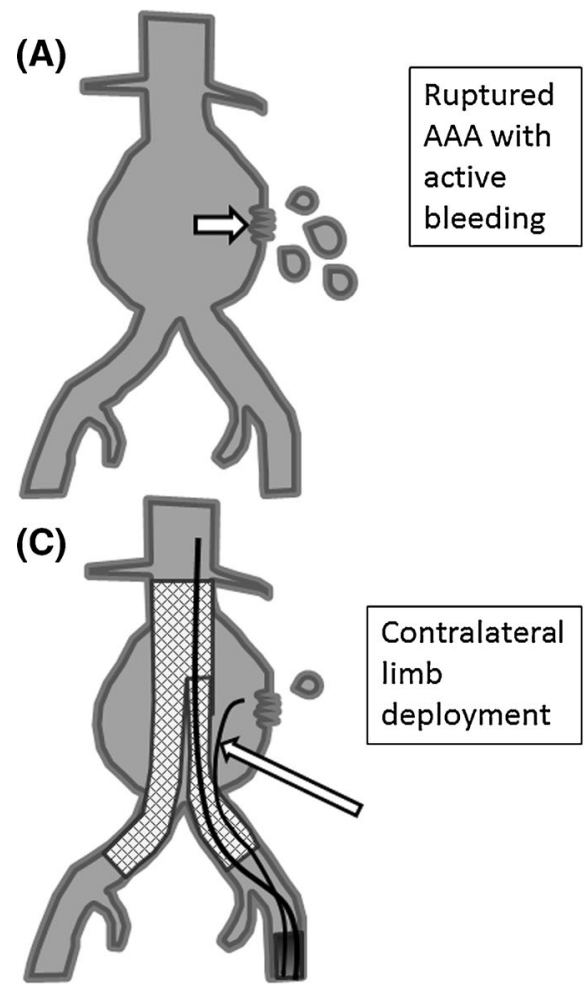

Fig. 1 Methodology of sac angiography in emergency EVAR in hemodynamically unstable patients with a ruptured AAA. A Active bleeding from the aneurysm sac was seen in a patient with a ruptured AAA. The white arrow indicates the bleeding site. B After a main body stent-graft was deployed, cannulation of the contralateral gate was performed. Another guidewire (white arrow) was then advanced into the sac of the aneurysm through the same sheath (black arrow) in

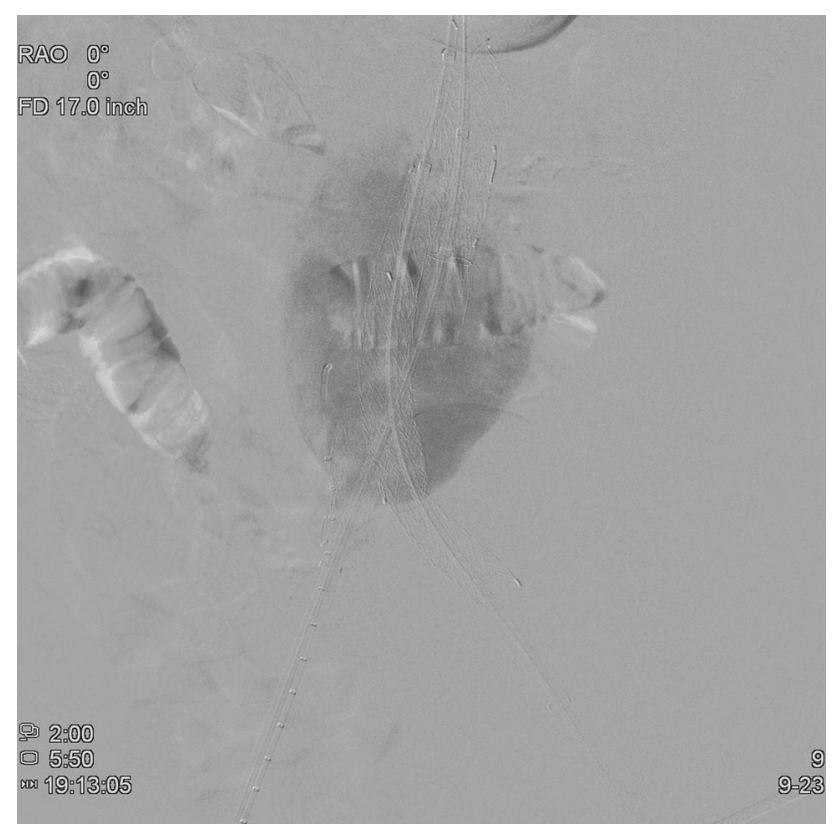

Fig. 2 Angiography within the sac of the aneurysm (sac angiography) revealed no active bleeding

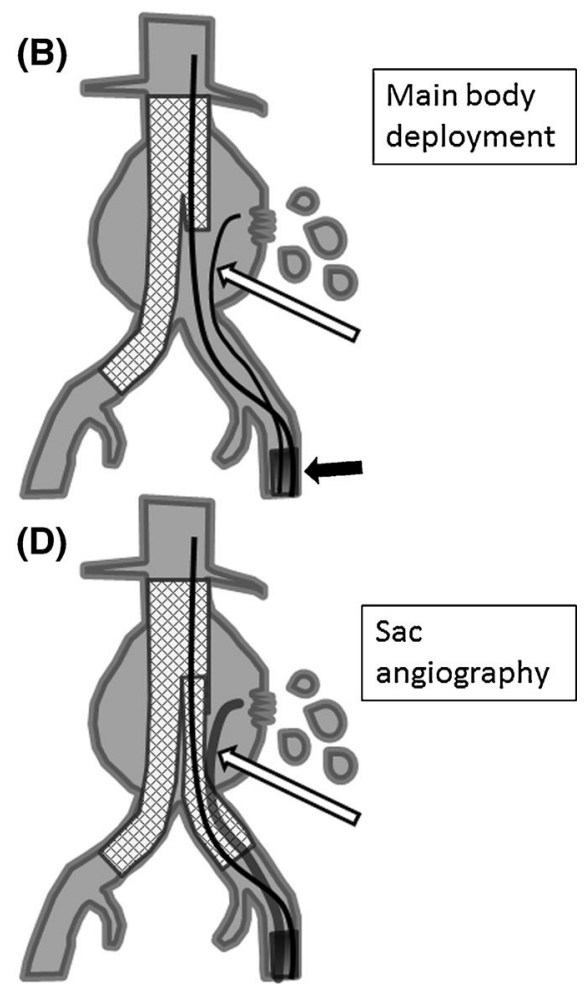

parallel. C While maintaining the position of the guidewire (white arrow), the contralateral limb stent-graft was deployed. D A 5-F catheter was advanced into the sac of the aneurysm onto the guide wire (white arrow). Angiography within the sac of the aneurysm (sac angiography) using the catheter was then performed to assess the presence or absence of active bleeding

the guidewire within the sac, the contralateral limb was introduced via the sheath onto the stiff wire and deployed (Fig. 1C). Next, a 5-F Cobra catheter (FANSAC; Terumo) was advanced into the sac of the aneurysm onto the standard J guidewire via the same sheath (Fig. 1D). Angiography within the sac of aneurysm (sac angiography) was then performed by manually injecting $10 \mathrm{ml}$ of contrast material through the Cobra catheter to identify the presence and site of active bleeding. When sac angiography revealed no active bleeding, glue embolization was not performed (Fig. 2). When sac angiography demonstrated active extravasation of the contrast material (Fig. 3B), a microcatheter (Wonder III; UTM Co. Ltd., Nagoya, Japan) and a 0.016-inch guidewire (AQUA V-III; Cordis, Miami Lakes, FL) were coaxially advanced into the ruptured hole of the aneurysm.

After confirming the position by selective angiography, embolization using a $50 \%$ mixture of $N$-butyl cyanoacrylate (NBCA) (B. Braun, Melsungen, Germany) and iodized oil (Lipiodol Ultra-Fluid; Terumo) was performed (Fig. 3C). Just before the NBCA mixture was injected, the 

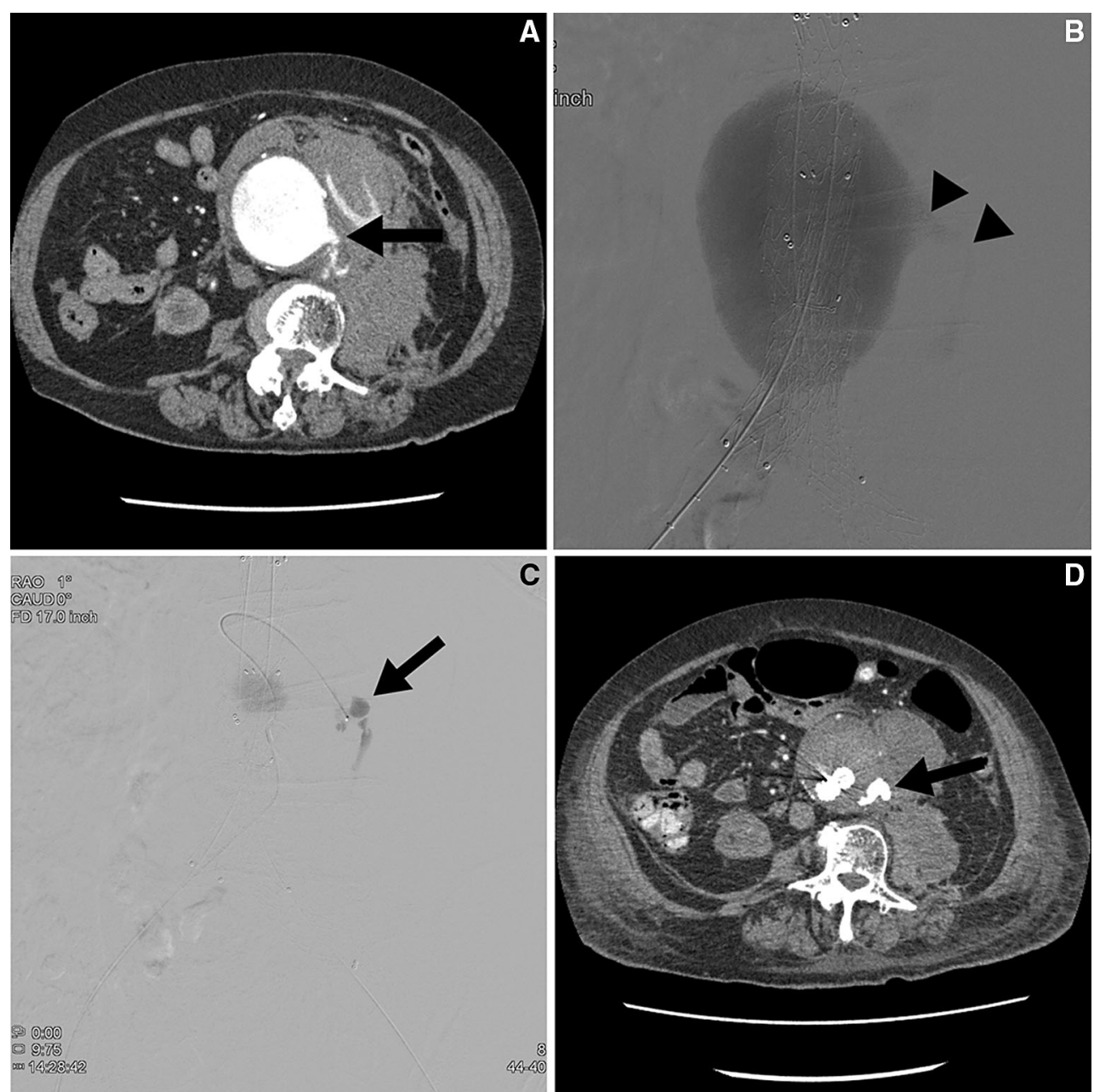

Fig. 3 Images of a hemodynamically unstable 80-year-old woman with a ruptured AAA. A Contrast-enhanced CT scan showed an infrarenal AAA and extravasation of the contrast material (arrow). B Sac angiography demonstrated active extravasation of the contrast material. The arrowheads indicate extravasation of contrast material

microcatheter was flushed with a $5 \%$ glucose solution to prevent polymerization within the microcatheter. The NBCA was injected slowly into the ruptured hole through a microcatheter, and the microcatheter was immediately retracted to prevent adherence of the catheter tip to the artery wall.

Finally, balloon dilation of the proximal neck, docking zone, and iliac legs was performed for adequate sealing of the stent-graft.

\section{Results}

Sac angiography was successfully performed in all three patients. Active bleeding from the aneurysm sac was observed in two patients (Patient 2 with Endurant and Patient 3 with Excluder), and subsequent embolization using an NBCA mixture was performed. In both cases, approximately

from the bleeding site. C The arrow indicates the NBCA injected through a microcatheter. D Contrast-enhanced CT scan at the 1.5month follow-up demonstrated accumulation (arrow) consistent with the active extravasation on preprocedural $\mathrm{CT}$ images

$1 \mathrm{ml}$ of a $50 \%$ mixture of NBCA was injected. No major intraoperative complications were encountered. Completion angiography revealed no evidence of active bleeding or type I or III endoleaks. The mean procedure time was 153 (range 110-189) min with a mean total fluoroscopic time of 31 (range 26-38) min. The mean transfusion volume was 8.6 (range 4-14) units of packed red cells and 5.3 (range 4-6) units of fresh-frozen plasma.

After the procedures, major complications were reported in all three patients: respiratory failure in two and renal insufficiency in one. After a mean hospital stay of 29 (range 11-45) days, all three patients were discharged.

During the follow-up (mean 8 months; range 6-12 months), CT angiography revealed no evidence of endoleaks or stent-related problems. An accumulation of the NBCA was recognized, consistent with the bleeding site (Fig. 3D). 


\section{Discussion}

In this technical report, we described the methodology of sac angiography in emergency EVAR for r-AAA and NBCA embolization to occlude the bleeding site in the aneurysm sac. Follow-up CT images demonstrated an accumulation of the NBCA consistent with the bleeding site, which indicated technical success.

Although EVAR in the treatment of r-AAAs seems to be feasible [1-3], it has a potential disadvantage associated with endoleaks, which could be undesirable in cases of active bleeding. Continuous bleeding via endoleaks may exacerbate hemorrhagic shock and abdominal compartment syndrome $[3,5]$.

To our knowledge, there are no reports on the performance of embolization of a bleeding site to achieve rapid hemostasis in a patient with r-AAA. To reduce the risk of type II endoleaks in elective EVAR, previous reports demonstrated intrasac "thrombization" using fibrin glue injection with or without the insertion of coils [6, 7]. However, because patients with r-AAA frequently also have severe coagulation abnormalities, NBCA seems to be a more feasible embolic material [8, 9]. In addition, a previous report described successful glue embolization of an r-AAA by a late type I endoleak after EVAR [10]. In the two cases, EVAR with NBCA embolization achieved prompt sealing with disappearance of the extravasation of contrast material as shown on the angiogram and an accumulation of NBCA as shown on the postoperative CT images. In emergent EVAR with active bleeding, we advocate that additional glue embolization is feasible.

The percentage of endoleaks after EVAR is generally reported to be 20-30\%, but endoleaks may be more common than previously believed $[11,12]$. That is, completion aortography sometimes fails to detect the presence of endoleaks. Therefore, we performed sac angiography to evaluate the presence or absence of active bleeding before aortography to detect the presence of endoleak. When the active bleeding continued, following embolization would achieve prompt hemostasis.

A potential complication associated with the use of NBCA is distal ischemic injury from nontarget glue embolization [10]. This risk can be minimized by coaxial microcatheterization of the rupture point after deployment of the stent-graft. However, this is associated with a risk of colonic or spinal cord ischemia [10, 13, 14].

In the two cases, either one or no bleeding site was demonstrated in the aneurysm sac by sac angiography. In patients with several bleeding sites in the aneurysm, embolization to fill the entire aneurysm sac may be required.

This small, retrospective series included patients who underwent sac angiography during emergency EVAR. Because patients who undergo r-AAA treatment are in severe conditions, it is difficult to demonstrate the feasibility of sac angiography and NBCA embolization of the bleeding site. Nonetheless, occlusion of the bleeding site in the aneurysm sac of the r-AAA in addition to the performance of EVAR may represent a possible technical improvement of conventional EVAR. Sac angiography would be of value as the basis for embolization of the bleeding site.

In conclusion, EVAR is associated with the potential risk of ongoing bleeding from type II or IV endoleaks into the disrupted aneurysm sac in patients with severe coagulopathy. Therefore, sac angiography and NBCA embolization during emergency EVAR may represent a possible technical improvement in the treatment of r-AAA in hemodynamically unstable patients.

Conflict of interest The authors declare that there are no conflicts of interest.

\section{References}

1. Ten Bosch JA, Teijink J, Willingendael E et al (2010) Endovascular aneurysm repair is superior to open surgery for ruptured abdominal aortic aneurysms in EVAR-suitable patients. J Vasc Surg 52:13-18

2. Brandt M, Walluscheck KP, Lajhnke T, Graw K et al (2005) Endovascular repair of ruptured abdominal aortic aneurysm: feasibility and impact on early outcome. J Vasc Interv Radiol 16:1309-1312

3. Mehta M, Darling RC III, Roddy SP et al (2005) Factors associated with abdominal compartment syndrome complicating endovascular repair of ruptured abdominal aortic aneurysms. J Vasc Surg 42:1047-1051

4. Berland TL, Veith FJ, Cayne NS, Mehta M, Mayer D, Lachat M (2013) Technique of supraceliac balloon control of the aorta during endovascular repair of ruptured abdominal aortic aneurysms. J Vasc Surg 57:272-275

5. Koike Y, Nishimura J, Nishimaki H et al (2013) Use of the Endurant stent graft system for ruptured infrarenal aortic aneurysms: short-term experience in nine patients. J Vasc Interv Radiol 24:1462-1469

6. Zanchetta M, Faresin F, Pedon L, Ronsivalle S (2007) Intraoperative intrasac thrombin injection to prevent type II endoleak after endovascular abdominal aortic aneurysm repair. J Endovasc Ther 14:176-183

7. Ronsivalle S, Faresin F, Franz F, Rettore C, Zanchetta M, Olivieri A (2010) Aneurysm sac "thrombization" and stabilization in EVAR: a technique to reduce the risk of type II endoleak. J Endovasc Ther 17:517-524

8. Kish JW, Katz MD, Marx MV, Harrell DS, Hanks SE (2004) $\mathrm{N}$-butyl cyanoacrylate embolization for control of acute arterial hemorrhage. J Vasc Interv Radiol 15:689-695

9. Yonemitsu T, Kawai N, Sato M, Tanihata H, Takasaka I et al (2009) Evaluation of transcatheter arterial embolization with gelatin sponge particles, microcoils, and $\mathrm{N}$-butyl cyanoacrylate for acute arterial bleeding in a coagulopathic condition. J Vasc Interv Radiol 20:1176-1187

10. Rusius VC, Davies RS, Hopkins JD et al (2011) Successful glue embolization of a late type Ia endoleak causing abdominal aortic aneurysm rupture. Vasc Endovasc Surg 45:198-201 
11. Lehmkuhl L, Andres C, Lücke C et al (2013) Dynamic CT angiography after abdominal aortic endovascular aneurysm repair: influence of enhancement patterns and optimal bolus timing on endoleak detection. Radiology 268:890-899

12. Habets J, Zandvoort HJ, Reitsma JB, Bartels LW, Moll FL, Leiner T, van Herwaarden JA (2013) Magnetic resonance imaging is more sensitive than computed tomography angiography for the detection of endoleaks after endovascular abdominal aortic aneurysm repair: a systematic review. Eur J Vasc Endovasc Surg 45:340-350
13. Bush RL, Lin PH, Ronson RS, Conklin BS, Martin LG, Lumsden $A B$ (2001) Colonic necrosis subsequent to catheter-directed thrombin embolization of the inferior mesenteric artery via the superior mesenteric artery: a complication in the management of a type II endoleak. J Vasc Surg 34:1119-1122

14. Jonker FH, Aruny J, Muhs BE (2009) Management of type II endoleaks: preoperative versus postoperative versus expectant management. Semin Vasc Surg 22:165-171 